in order to define the problems in the process of weaning in breastfed babies.

Methods Sample of the study was consisted with 86 volunteer mothers who registered to 3 different family health center in center of Sakarya province and who have 2 years old children left the breast milk. The data were collected using a questionnaire developed by researchers. The data were analysed using a statistical packet programme.

Results The mean age of the mothers was $29.52 \pm 5.49$. $50.6 \%$ $(n=44)$ of mothers were primary school graduates. $52.9 \% \quad(n=46)$ of families have described to their economic situation as moderate. $47.1 \%(n=41)$ of the mothers $7-12$ months, $27.6 \%$ of $(n=24)$ 0-6 months, $19.7 \%(n=17) 13-24$ months, $5.7 \%$ of $(n=5)$ in 25 months reported weaned the baby. $73.5 \%(n=64)$ of the mothers cut the breastfeeding slowly was determined. Problems experienced by mothers at the beginning of the process of weaning were found as pain in the chest and swelling $(20.7 \% \mathrm{n}=18)$ and maternal stress, anxiety and indecision $(12.6 \% n=11) .39 \%$ of children $(n=34)$ crying, restlessness, irritability and a change in temperament, $12.6 \%(n=11)$ reduction in body weight gain, $8 \%(n=7)$ frequent illness, $2.3 \%(\mathrm{n}=2)$ sleep pattern disorder were reported by their mothers.

Conclusion In the study, various problems occured in children and mothers were found in the process of weaning.

\section{MOTHERS' PERCEIVED FAMILY SUPPORT AND AFFECTING FACTORS IN POSTPARTUM PERIOD}

doi:10.1136/archdischild-2012-302724.1869

N Çınar, D Köse, S Altınkaynak. Department of Child Health Nursing, Sakarya University School of Health Sciences, Sakarya, Turkey

Background and Aim Having a new baby, mothers need the support of relatives due to they often live stress or sometime faced with difficult situations. The study was done as descriptive in order to determine mothers' perceived family support and affecting factors in postpartum period.

Methods Sample of the study was consisted with 140 volunteer mothers in Sakarya. The data were collected using a questionnaire developed by researchers and "Perceived Social Support from Family Scale" (PSS-Fa) developed by Procidana and Hellen (1983) and also validity and reliability made by Eskin (1993).

Results Most of the mothers were determined as $75.7 \%(n=106)$ between the ages of $21-35,87$ and $48.6 \%(n=68)$ primary school graduates. The mean total score of the scale was determined as 14.04 \pm 4.29 ( $\min =1, \max =20)$. When compared a total score received from the scale with the economic situation of families; Family support were determined as higher $(\mathrm{F}=16.014 \mathrm{p}=0.000)$ in families with moderate and good economic situation compared to the bad economic situation. Between perceived family support and the number of children showed a significant relationship ( $F=3.462$ $\mathrm{p}=0.010$ ). It is determined that when the number of chilren was increased family support was reduced. Relationship between mother's age, employment status and perceived family support were determined as not statistically significant (respectively $\mathrm{F}=.554$ $\mathrm{p}=0.576, \mathrm{t}=0.335 \mathrm{p}=0.738$ ).

Conclusion Mothers in bad economic situation and having more number of children found low perceived family support is remarkable case.

\section{ASSESSING THE INFORMATION AND SUPPORT NEEDS OF PARENTS OF YOUNG CHILDREN IN CANADA}

doi:10.1136/archdischild-2012-302724.1870

${ }^{1} \mathrm{MA}$ Devolin, 'D Phelps, ${ }^{2 \mathrm{~T}}$ Duhaney, ${ }^{3 \mathrm{~K}}$ Benzies, ${ }^{4} \mathrm{C}$ Hildebrandt, ${ }^{4} \mathrm{~S}$ Rikhy, ${ }^{5} \mathrm{~J}$ Churchill,

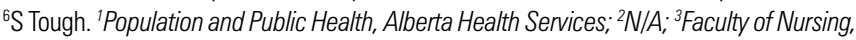

University of Calgary; ${ }^{4}$ Alberta Children's Hospital; ${ }^{5}$ Public Health, Calgary Zone, Alberta Health Services; ${ }^{6}$ Department of Paediatrics, University of Calgary, Calgary, AB, Canada

Aim To determine the parenting information and support needs of parents of young children in an urban center in Canada.

Methods A self-administered survey was mailed to a stratified random sample of 1064 parents of children aged 6 years and under. The survey asked about parents' preferred sources of support, information needs, sources and modes of information delivery and perceived barriers to accessing parenting support services.

Results A $33.7 \%$ response rate was obtained ( $n=359$ surveys). Breastfeeding, car seat safety, caring for a new baby, supporting their child's development and sleep issues were considered 'somewhat' and 'very' important by more than $95.8 \%$ of respondents. Informal sources of support were rated as more important and more valuable than formal supports. The internet, drop-in programs for parents and children, books, organized playgroups, and classes/ information sessions, were identified as the most preferred ways to access parenting information. Parents reported a lack of knowledge and awareness of programs, lack of time, lack of child care, and offered at inconvenient times, as the top barriers to attending parenting services and supports.

Conclusions In general, parents want information on early childhood development and parenting. This assessment contributes to the limited information on the unique needs of parents with children 0-6 years in Canada. The results have important implications for the planning, delivery and dissemination of future parenting education resources, programs and services, namely creating opportunities for social networking and exploring on-line methods for information delivery.

\section{INTERVENTION PROJECT: INTEGRATION OF MATERNITY CARE INTO THE NEONATAL INTENSIVE CARE UNIT, KEEPING MOTHER AND INFANT TOGETHER DIRECTLY AFTER BIRTH}

doi:10.1136/archdischild-2012-302724.1871

L Fredbo-Nielsen. Neonatal Intensive Care Unit, Rigshospitalet, København Ø, Denmark

Background When an infant is born at Rigshospitalet in Denmark, and is transferred to NICU, the bonding between infant and mother is complicated because the mother is transferred to the maternity ward. With the existing knowledge of the infant's congenital competencies to create and preserve relations, the close relation to the mother is necessary for the future development. The competencies includes signals for the infant such as eye contact, crying, sucking on moms breast, smell, rate during communication between mother and infant and smile. A positive and secure bonding predisposes feelings such as basic security, intensity and devotion. Failure and insecure bonding can lead to violent negative feelings. International research shows the benefit of family centered care and skin-to-skin contact for preterm babies and their parents for e.g. temperature, weight gain, sleep, breastfeeding, and bonding.

Aims To facilitate the bonding between the newborn ill infant and the mother.

Methods Transfer of the mother (after normal delivery or 4 hours after planned caesarean) directly to the NICU with her infant.

Two hours training of all 120 neonatal nurses in obstetric nursing. Questionnaire to the mothers.

Results 58 mothers have been transferred directly from the delivery room to NICU. Results from the questionnaire to the mothers will be available on the poster.

Discussion Is it possible for a nurse trained in NICU to take care of the obstetric mother?

Is the environment at NICU acceptable for both mother and infant? 


\section{AN INVESTIGATION ON THE STATUS OF TERM PREMATURE AND LOW BIRTH WEIGHT AND ITS ASSOCIATION WITH SOME MATERNAL FACTORS}

doi:10.1136/archdischild-2012-302724.1872

PT Taheri. Pediatric Nursing Department, Isfahan University of Medical Sciences and National Children's Research Centre, Isfahan, Iran

Introduction The cause of this mortality is either being premature or low birth weight of newborns and congenital diseases. Provision of health for this most precious generation is the main duty of each nation and government. Health promotion and the right of newborn's for living, Pregnancy development, delivery prognosis and newborn's survival all come true just by maternal health.

Materials and Methods This is a descriptive comparative, one step, three group study, The population studied comprised 380 term, premature and low birth weight newborn's in hospitals, post (maternity delivery), newborn and NICU wards selected target basedly from selected university hospitals in Isfahan.

Results Term, premature and low weight newborn's status was questioned from the mother, investigated in the infants' files and then was recorded. The findings showed that $57.1 \%$ of the infants were boys and $46.6 \%$ girls.

Mothers mean age based on new borns' birth weight through spearman correlation test shows a non significant association between birth weight and mothers' age, $(\mathrm{r}=\% 2 \mathrm{P}=0.698)$. (Analyzing) Mothers' mean age pre pregnancy based on infants' weight status through speasrman correlation test shows a non significant association between infants' birth weight and pre pregnancy mothres' weight ( $\mathrm{p}=0.883$ ).

Discussion The role of mothers' care in maternal care get more remarkable. Meanwhile, the health providing team maternal care.

\section{BREASTFEEDING PROBLEMS DURING FIRST THREE POSTNATAL DAYS}

doi:10.1136/archdischild-2012-302724.1873

${ }^{1} \mathrm{~J}$ Raju, ${ }^{1} \mathrm{M}$ Vatsa, ${ }^{2} \mathrm{RK}$ Agarwal. ' College of Nursing; ${ }^{2}$ Department of Pediatrics, All India Institute of Medical Sciences, Delhi, India

Background and Aims We surveyed late preterm and term mother-newborn dyads $(\mathrm{n}=380)$ in a tertiary care hospital to determine the epidemiology of breastfeeding problems during initial 72 hours.

Methods The subjects were enrolled within 12 hours of delivery and assessed 12 hourly for perception of and documented breastfeeding problems using validated open ended questionnaire and checklist respectively.

Results A total of 220 (57.9\%) mothers delivered vaginally, and $228(60 \%)$ had no previous experience of breastfeeding. Nearly all mother-infant dyads had one or more breastfeeding related problems. The major self reported problems included insufficiency of milk (341, 89.7\%), difficulty in latching $(145,38.2 \%)$ and breast related problems $(112,29.5 \%)$. The major documented problems included poor attachment $(378,99.5 \%)$, poor positioning (378, $99.5 \%)$, breast engorgement $(76,20 \%)$ and poor sucking $(65,17.5 \%)$. A significant proportion of mother-infant dyads continued to have breastfeeding problems at 72 hours after delivery. The common problems were poor positioning $(121,31.8 \%)$, poor attachment $(121,31.8 \%)$ and breast engorgement $(71,18.7 \%)$. Caesarean delivery was found to be a significant predictor of self reported breastfeeding problems.

Conclusion There is a huge burden of breastfeeding problems in mothers delivering at gestation of 34 weeks or more. There is a need to examine the impact of breastfeeding problems and the interventions directed to them on breast feeding outcomes.

\section{CONTRIBUTION TO INTERCULTURAL ADAPTATION OF THE “NURSE PARENT SUPPORT TOLL” TO PORTUGUESE REALITY}

doi:10.1136/archdischild-2012-302724.1874

${ }^{1} \mathrm{AP}$ França, ${ }^{2} \mathrm{SM}$ Valadão, ${ }^{1} \mathrm{MC}$ Reisinho. ${ }^{1}$ UNIESEP (Oporto College of Nursing Resaearch Unit), Oporto College of Nursing, Porto; ${ }^{2}$ Unidade de Cuidados Especiais Pediátricos/Neonatais, Hospital Santo Espírito de Angra do Heroísmo-EPER, Angra do Heroísmo, Portugal

The hospitalization of a child at risk appears to be an ongoing source of stress for parents and several studies highlighted the importance of nurses' support. Therefore, the existence of a scale that allows parents to assess nurses' support, will contribute to build a more effective relationship between them, in order to provide excellence in nursing care, not only directed to the child but also to the family, contributing to increased parental satisfaction related to nursing care.

It's a methodological study that took place in a portuguese neonatal and pediatric intensive unit, that intends to make the first contribution to intercultural adaptation of the scale "Nurse Parent Support Tool" for the European Portuguese and to Portuguese culture, aiming at its future use as a measure of the perception that parents have about the support they receive from nursing staff during hospitalization of their child.

Guaranteeing the semantic and conceptual equivalence involved the following stages: 1- Translations, 2- Synthesis of translations, 3- Back-translation and 4- Synthesis of back-translation. The evaluation of its content validity involved: Stage 5 - Review by the Expert Committee, and stage 6- Pretest.

The Portuguese version of NPST kept the conceptual and semantic equivalence, as the original, and showed good levels of content validity in Portuguese culture.

Results justify future investigations to evaluate other psychometric properties and thus make it scientifically valid, for implementation in clinical practice of portuguese pediatric nursing.

\section{KANGAROO MOTHER CARE IN A NEONATAL CONTEXT: PARENTS' EXPERIENCES OF INFORMATION AND COMMUNICATION}

doi:10.1136/archdischild-2012-302724.1875

'D Lemmen, ${ }^{2 P}$ Fristedt, ${ }^{3} \mathrm{~A}$ Lundqvist. ${ }^{1} B I V A$, Skånes Universitets Sjukhus, Lund; ${ }^{2} B V C$ Capio Citykliniken, Landskrona; ${ }^{3}$ Dep of Health Sciences, Lund University, Lund, Sweden

Kangaroo Mother Care (KMC) is an evidence-based nursing practice with many benefits for children and parents. The purpose of this study was to describe mothers' and fathers' experiences of information and communication from the staff in connection with KMC and their experiences during the application of KMC. A qualitative study with semi-structured interviews was performed. The sample consisted of 12 families $(n=20)$. The results show that the information and communication was experienced as both optimal and suboptimal including following themes: safe and confusing, satisfactory and unsatisfactory and enhanced self-esteem and lack of self-esteem. The experiences during application of KMC included belonging and alienation as well as happiness and anxiety. The overall theme showed that information and communication of $\mathrm{KMC}$ is both crucial and not crucial for parents to experience KMC as optimal nursing care. The conclusion is that staff in neonatal units, where the KMC is implicated, should review their practices regarding the process of information and communication between parents and staff. This requires further research into the implementation of communication theories for the staff in neonatal units in a satisfactory way for the parents. BEING THE PARENT OF A BABY IS LIKE SCALE

doi:10.1136/archdischild-2012-302724.1876 